\title{
DESCRIPCIÓN HISTOLÓGICA DEL COMPLEJO FOLICULAR PILOSO EN CRÍAS DE ALPACAS
}

\author{
Histological Description of the Hair Follicle in the Young Alpaca
}

Elmer Badajoz L. ${ }^{1}$, Nieves Sandoval Ch. ${ }^{1,2}$, Wilber García V. ${ }^{3}$ y Danilo Pezo C. ${ }^{3}$

\section{Resumen}

\begin{abstract}
El presente estudio tuvo el propósito de caracterizar la distribución y asociación de los folículos que conforman la piel de la alpaca. Se tomaron muestras de piel de 42 crías de alpacas Suri y Huacaya, de ambos sexos, y diversos colores de manto. Las muestras se colectaron con sacabocado de la zona del costillar medio y fueron procesadas mediante técnicas histológicas estándares, utilizando la coloración H y E. Los folículos pilosos se encuentran formando nidos foliculares, distribuidos como grupos foliculares compuestos (GFC) y grupos foliculares simples (GFS). Los primeros se encuentran conformados por folículos primarios rodeados de folículos secundarios, mientras que los GFS solo están conformados por folículos secundarios. Además, los folículos primarios pueden ser solitarios, denominados folículo primario extra grupo folicular (FPEGF) o formando parte de un grupo folicular compuesto denominado folículo primario intra grupo folicular (FPIGF). Los grupos foliculares están conformados uno o más folículos primarios y un número variable de folículos secundarios; sin embargo, en las alpacas Suri se observa un trío folicular primario, caracterizado por presentar un folículo primario central y dos laterales. Las características de asociación y distribución de los folículos, así como de su citoarquitectura permiten establecer la presencia del denominado "Complejo Folicular Piloso" en la piel de las alpacas.
\end{abstract}

Palabras clave: alpaca, cría, piel, folículo piloso

\section{AbSTRACT}

The present study was carried out to characterize the distribution and an association of hair follicles of alpaca skin. Samples were collected from 42 young Suri and Huacaya alpacas, of both sexes and with various hair colours. Samples were collected by punch skin biopsy in the middle costal zone and processed for histological study using H-E staining. Hair follicles formed follicular nests distributed as a compound follicle group (CFG) and simple follicle group (SFG). The first was composed of primary follicles surrounded by secondary follicles, while that latter was only composed of secondary follicles. Furthermore, primary follicles can be solitary and named Extra Primary Follicle

${ }^{1}$ Laboratorio de Histología, Embriología y Patología Veterinaria, ${ }^{3}$ Estación Experimental del Centro de Investigación IVITA-La Raya, Universidad Nacional Mayor de San Marcos, Lima

${ }^{2}$ E-mail: nsandovalc@unmsm.edu.pe 
Follicular Group (EPFFG) or forming compound follicle groups named Internal Primary Follicle Follicular Group (IPFFG). Follicular groups are formed by one or more primary follicles and a variable number of secondary follicles; however, in the Suri alpaca, was observed groups of three primary follicles, one larger and central and two smaller at each side. The characteristics of association and distribution of follicles and the cytoarchitecture allowed pointing out the presence of a Hair Follicular Complex in the alpaca skin.

Key words: alpaca, offspring, skin, hair follicle

\section{INTRODUCCIÓN}

La piel protege al cuerpo de los agentes térmicos, mecánicos, químicos y microbiológicos, mediante su epidermis cornificada, donde se encuentran varios tipos de fibra, pelos y glándulas de secreción externa (Junqueira y Carneiro, 1988; Banks, 1993). El estudio de los folículos de la piel de los mamíferos, especialmente en ovinos, ha sido seguido con interés desde el siglo pasado, encontrándose cambios en las diversas etapas de crecimiento, factores que podrían tomarse en cuenta en los programas de selección con fines de aumentar la producción de lana por animal (Carter y Clarke, 1957).

En un programa de selección por fibra, la estructura folicular de la piel representa uno de las factores de importancia, como es el caso del plan de selección de los ovinos merinos superfinos y en el programa de selección de la alpaca australiana (Charry, 1998). Se han realizado estudios de los folículos pilosos de la alpaca en etapas embrionales y adultas (Tapia, 1969; Mamani, 1988), pero solo se tiene informaciones preliminares de las estructuras foliculares de la piel (Gaitan, 1967). Por lo tanto, el presente estudio tuvo como objetivo efectuar una descripción sobre la caracterización, distribución y asociación de los folículos que conforman la piel de las alpacas, a fin de servir como fuente de información para una eficiente selección de reproductores a temprana edad.

\section{Materiales y Métodos}

Se emplearon 21 crías de alpacas de la variedad Huacaya y 21 crías de la variedad suri, de ambos sexos y de 6 a 10 meses de edad, pertenecientes a la Granja de Camélidos La Raya de la Estación Experimental del Centro de Investigación IVITAMaranganí, Cusco.

Se tomaron muestras de piel de la zona del costillar medio, mediante cortes de forma circular con un sacabocado de $6 \mathrm{~mm}$ de diámetro. Las muestras se colocaron en frascos conteniendo una solución fijadora de formol al 15\%, y se trasladaron al Laboratorio de Histología, Embriología y Patología de la Facultad de Medicina Veterinaria de la Universidad Nacional Mayor de San Marcos, Lima, para su procesamiento.

Las muestras se dividieron en dos cortes, uno perpendicular a la superficie de la piel y el otro se destinó para obtener secciones paralelas a la superficie de la misma. Ambos cortes se sometieron al proceso histológico convencional de deshidratación, clarificado, inclusión en parafina, cortes al micrótomo, montaje en láminas, desparafinado, y coloración con Hematoxilina - Eosina. En las secciones o cortes perpendiculares a la superficie de la piel y transversales a los folículos pilosos se identificaron los nidos o grupos foliculares. Paralelamente, se caracterizó el tipo de folículos que confor- 


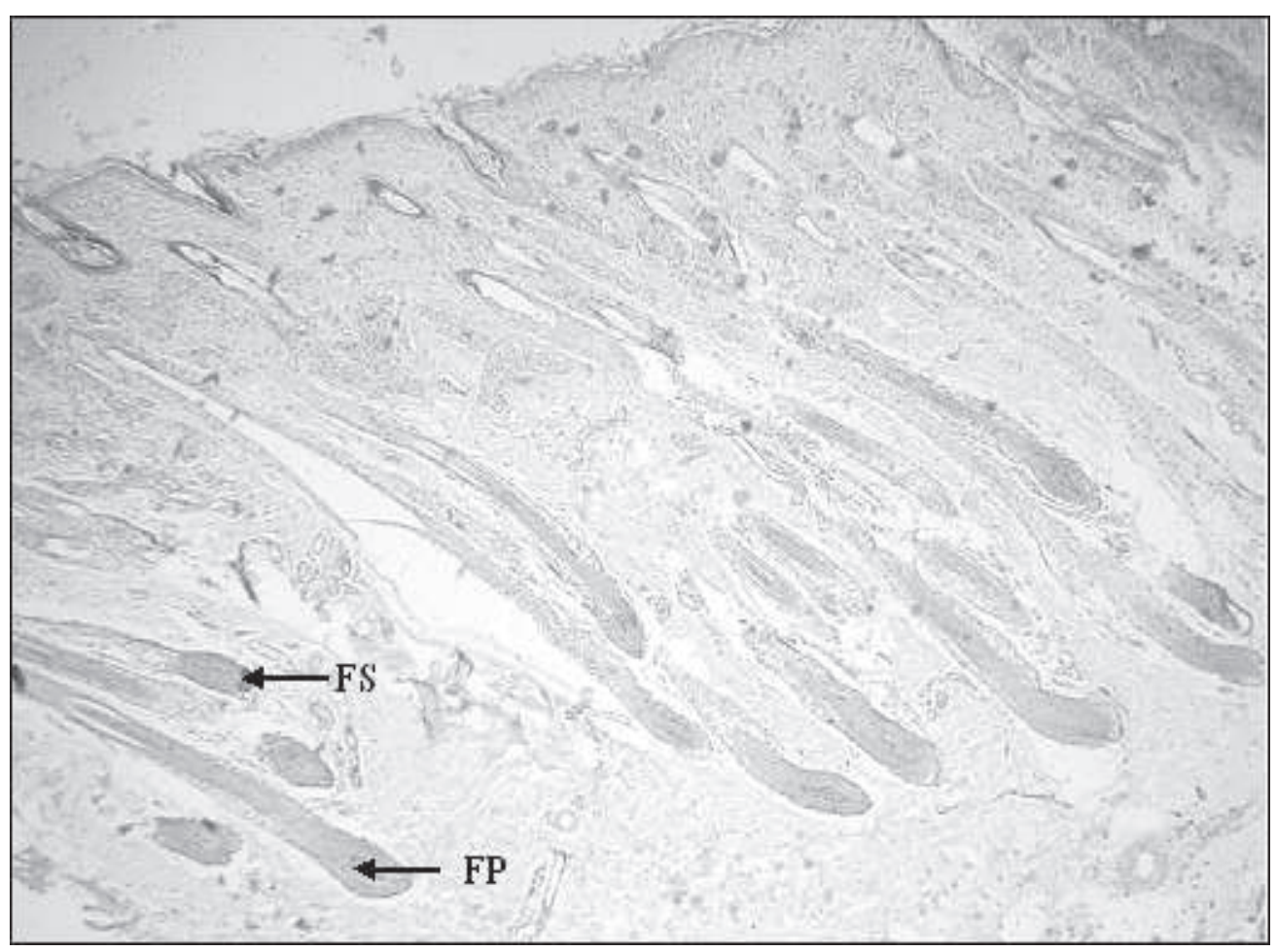

Figura 1. Corte perpendicular a la superficie de la piel de alpaca. Coloración HE (50X). $F P=$ Folículo primario, $F S=$ Folículo secundario

man cada grupo folicular a una magnificación de $400 \mathrm{X}$ y $100 \mathrm{X}$.

\section{Resultados}

La sección perpendicular a la superficie de la piel evidencia que los folículos pilosos suelen estar colocados oblicuamente en un ángulo de 30 a 60 grados con respecto a la epidermis. La emergencia de los folículos hacia la superficie es muy singular en esta especie, ya que varios folículos secundarios medulados y no medulados más un folículo primario, emergen a la superficie por un mismo orificio, mientras que los folículos primarios de mayor profundidad emergen en forma individual (Fig. 1).

La sección paralela a la superficie epidérmica de la piel de ambas variedades de alpacas evidencia a los folículos pilosos en su gran mayoría formando nidos foliculares (Fig. 2), los cuales se encuentran distribuidos en su mayor parte como grupos foliculares compuestos (GFC), y en menor proporción como grupos foliculares simples (GFS). Los primeros se encuentran conformados por folículos pilosos primarios (FP) y folículos secundarios (FS), delimitados completamente por tejido conectivo denso que se infiltra entre los FS y el FP, formando un fino estroma conectivo (Fig. 3). Por otro lado, se observa que los GFS carecen de folículos primarios, conformados solamente por FS, que se encuentran mayormente fusionados a través de su vaina radicular externa (VRE) (Fig. 4). Asimismo, los FS de la variedad Suri se presentan usualmente asociados a un acino de glándula sebácea.

El FP dentro de un nido folicular se caracteriza por encontrarse asociado a una glándula sudorípara apocrina (Fig. 5). El FP 


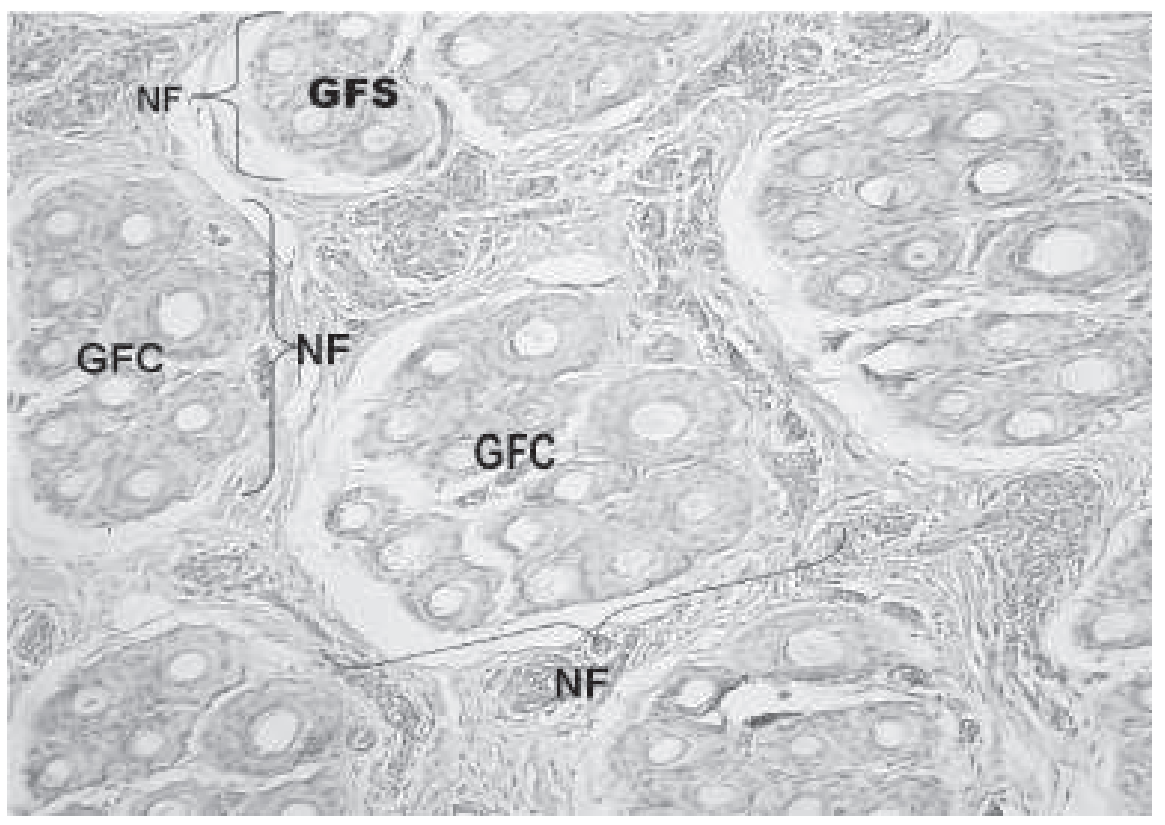

Figura 2. Distribución de los nidos foliculares (NF) como grupos foliculares compuestos (GFC) y grupos foliculares simples (GFS) en un corte paralelo a la superficie de la piel de alpaca. Coloración HE, $100 X$.

tiene un mayor diámetro con respecto a los FS y su fibra o tallo piloso presenta una médula de forma ovoide. Por otro lado, los FS secundarios que conforman un GFC presentan fibras o tallos de pelo con una medulación variable, caracterizada por nidos heterógeneos conformados por folículos con fibra medulada y no medulada, especialmente en la variedad Suri (Fig. 6). En algunos cortes, las alpacas Huacaya presentan FS con fibras ameduladas y rodeados por un tabique conectivo sumamente capilarizado conformado por paquetes de plexos tortuosos.

La estructura y el tamaño de los grupos foliculares compuestos, así como el tamaño del tipo de folículos que lo conforman, varía ampliamente entre las dos variedades. Así se tiene que los GFC están conformados predominantemente por un FP rodeado de un número variable de $\mathrm{FS}$, aunque hay GFC constituidos por 2 y 3 FP (Fig. 7); sin embargo, en los cortes de alpacas Suri se hace evidente la observación del trío folicular primario, caracterizados por presentar un FP central y dos FP laterales, los cuales presentan su propio conducto sudoríparo (Fig. 8).
El número de FS rodeando a un FP dentro de un GFC fue de 3 a 20, en alpacas Suri, y de 3 a 26 en Huacayas. El tamaño de los nidos foliculares en ambos grupos de alpacas es muy variable, observándose nidos pequeños, intermedios y grandes, los cuales se encuentran rodeados por una cubierta de tejido conectivo denso, donde se halla el conducto de la glándula sudorípara, arteriolas y capilares. El grosor del tejido conectivo depende de la densidad de los nidos foliculares; es decir, a mayor densidad folicular, menor estroma conectivo entre nidos foliculares.

En los cortes transversales se visualiza la existencia de folículos cerca a la superficie de la epidermis, observándose en el interior estructuras compatibles con fibras, pudiendo haber hasta tres, semejantes a "penachos" (Fig. 9).

Los folículos pilosos primarios distribuidos de manera individual fuera del GFC se han denominados folículos primarios extra grupo folicular (FPEGF) para los fines de este estudio. Estos contienen una estructura 

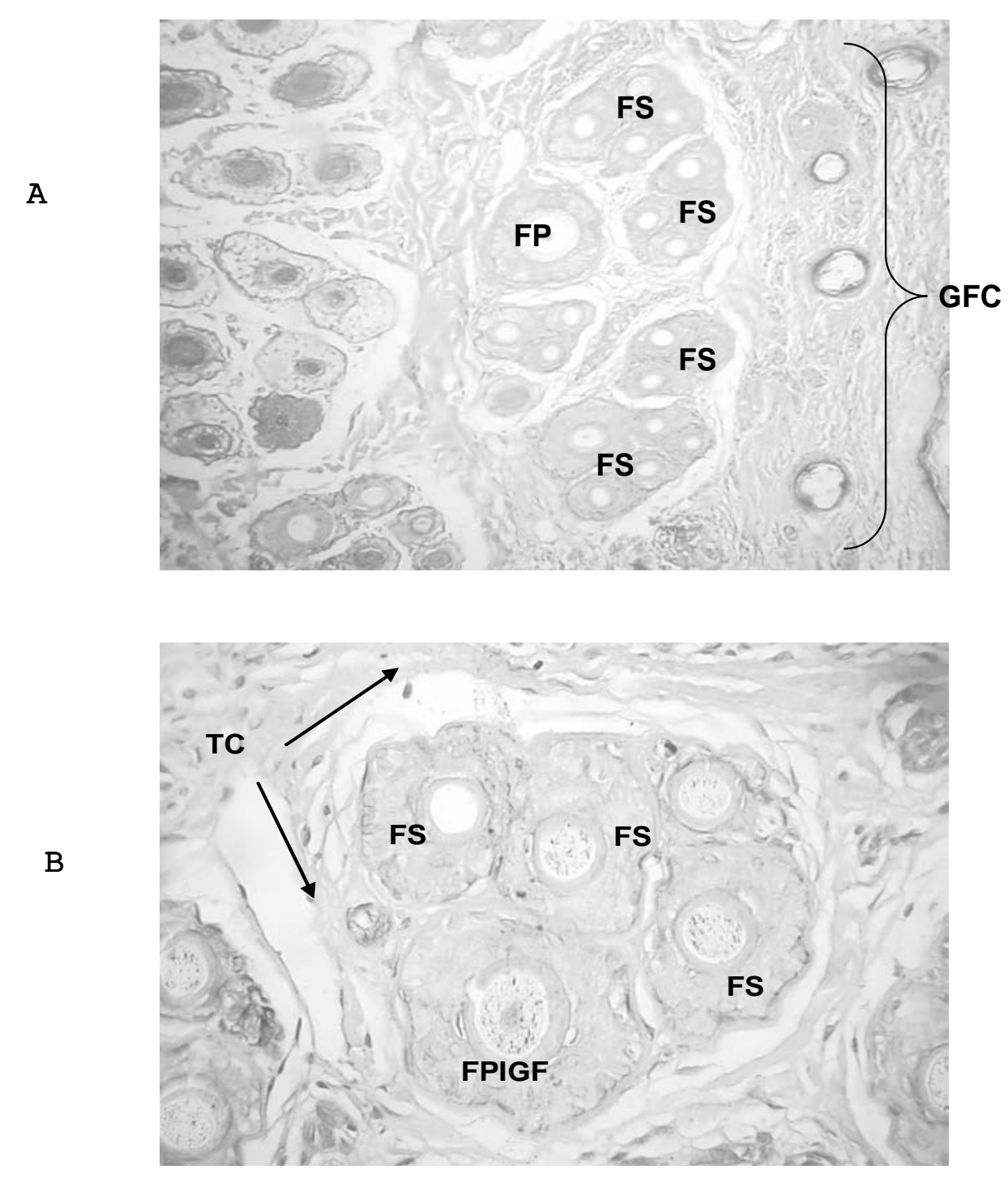

Figura 3. Grupo folicular piloso de la alpaca. A) Estructura del grupo folicular compuesto (GFC) en alpaca Suri. $F P=$ Folículo primario $F S=$ Folículo secundario. Coloración $H E(100 X)$. B) Estructura predominante del grupo folicular en alpaca Huacaya. TC $=$ Tejido conectivo dérmico, FPIGF = Folículo primario intra grupo folicular, $F S=$ Folículo secundario. Coloración HE. 400X.

radicular bien desarrollada, así como un mayor diámetro de fibra que evidencia una prominente médula eosinófilica, caracteres que definen al pelo (Fig. 10). Estos son escasos y se encuentran en la mayoría de cortes, adyacentes al nido folicular y rodeado por una vaina de raíces fibrosas de tejido conectivo. Se presentan en ambos grupos de alpacas, pero en el grupo Huacaya, la médula es más prominente ocupando casi la mitad del diá- metro de la fibra en relación a la corteza y tiene forma arriñonada (Fig. 11), mientras que en los Suri es menos prominente y tiene forma ovoide.

Al corte transversal, el pelo en el FPEGF presenta de adentro hacia afuera las siguientes estructuras: La médula, que es grande y ocupa, aproximadamente, la mitad de la fibra, presentando generalmente la for- 


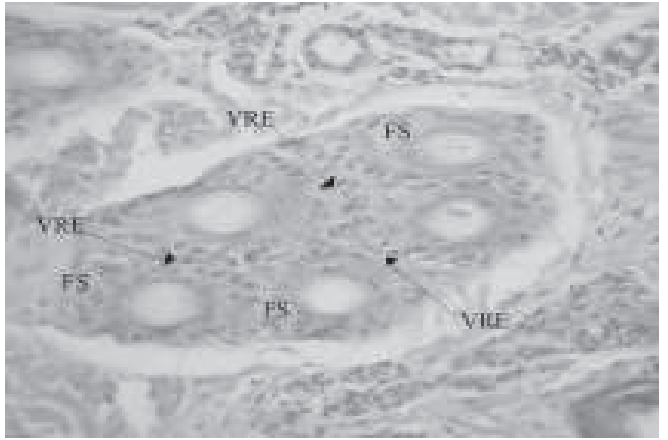

Figura 4. Grupo folicular simple mostrando fusión de la vaina radicular externa (VRE) (flechas) de los folículos secundarios Coloración HE, $400 X$

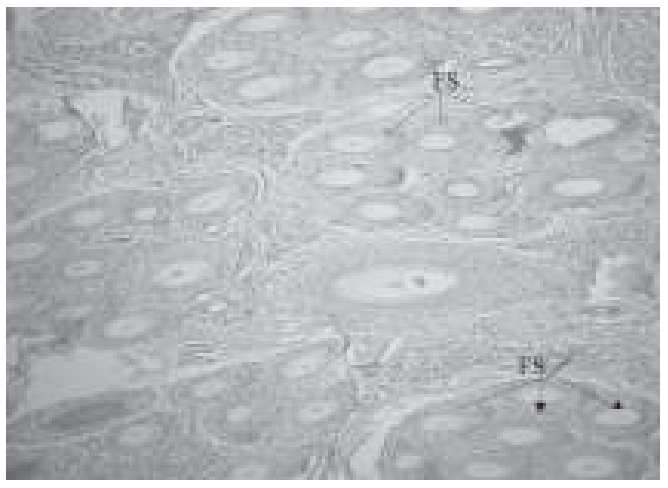

Figura 6. Folículos secundarios (FS) mostrando variable medulación en alpaca Suri. Coloración HE, $400 X$

ma de una hélice incompleta o arriñonada de color acidófilo con la coloración HE; la corteza, que se presenta de aspecto hialino alrededor de la médula; y la cutícula de la fibra, que es la capa más superficial de la fibra y limita a la misma. La vaina radicular interna (VRI) presenta tres capas: una fina capa cuticular de aspecto brillante, la capa de Huxley constituida por 2 a 3 capas de células cúbicas acidófilas y la capa de Henle, constituida por una capa de células acidófilas de aspecto homógeneo, brillante y rica en

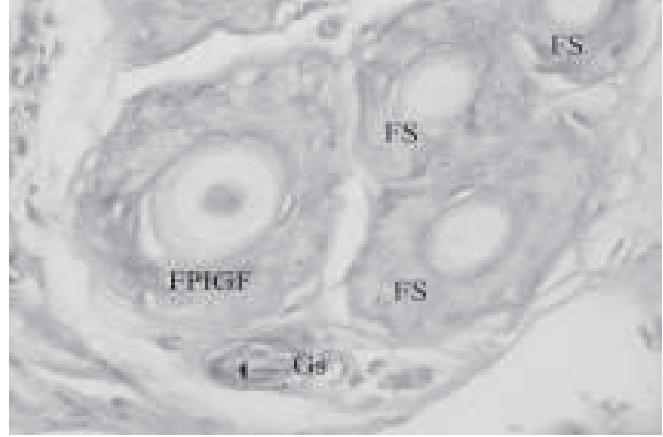

Figura 5. Diferenciación histológica del folículo primario intragrupo folicular (FPIGF) asociado a una glándula sudorípara (Gs). $F S=$ Folículo secundario. Coloración $H E$, $400 X$

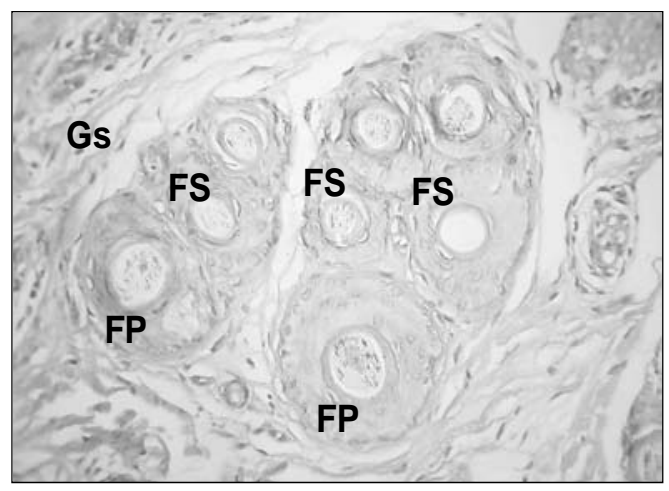

Figura 7. Doble folículo primario (FP) rodeado de varios folículos secundarios (FS) en grupo folicular de alpaca Huacaya. $G s=$ Glándula sudoripara. Coloración HE, $400 X$

queratina. La VRE está conformada por 4 a 5 capas de células grandes similares a las células de la epidermis. La vaina conjuntiva que rodea al folículo se encuentra más compacta alrededor del mismo y puede estar acompañado de una glándula sebácea, y/o un conducto de la glándula sudorípara.

En el caso del folículo primario intra grupo folicular (FPIGF), la fibra del folículo piloso primario, en el corte transversal, presenta de adentro hacia afuera las siguientes estruc- 


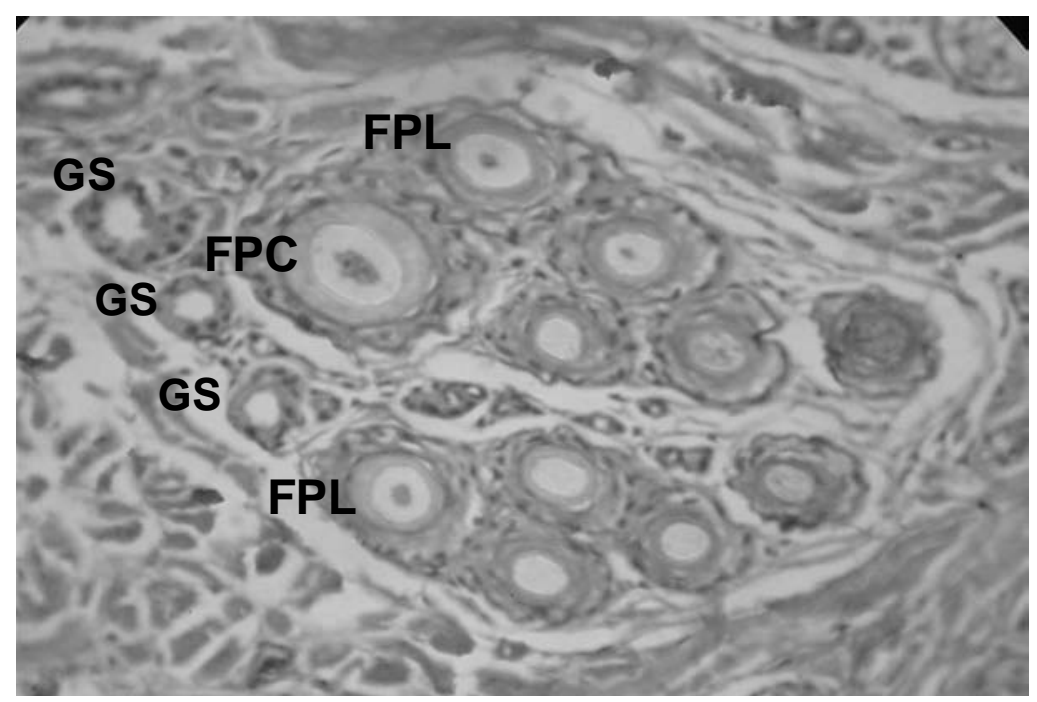

Figura 8. Trío folicular primario en Grupo Folicular compuesto de alpaca Suri. FPC = Folículo primario central, $\mathrm{FPL}=$ Folículo primario lateral, $\mathrm{Gs}=$ Glándula sudorípara. Coloración $H E, 400 X$

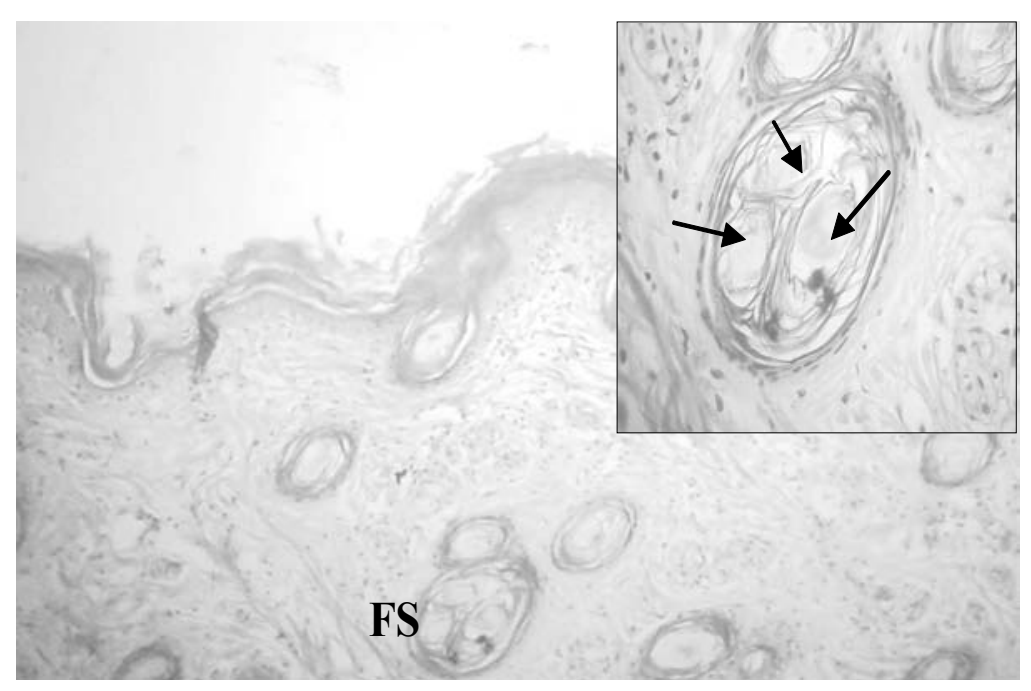

Figura 9. Penachos de fibras en piel de alpaca: folículos secundarios (FS) conteniendo más de una fibra en su interior (recuadro superior derecho, flechas). $H E, 100 X$

turas: la médula, que se aprecia como una demarcación circular u ovoide de manera variable; la corteza, queratinizada de aspecto hialino y homogéneo que se prolonga a lo ancho de la fibra; y la cutícula, que se encuentra delimitando a la fibra. La VRI está formada por una capa constituida por escasas células en donde la capa cuticular más interna se muestra muy delgada, mientras que la capa media denominada Huxley y la capa más externa (Henle) constituyen una zona que se aprecia como una franja circular hialinizada. Por otro lado, la VRE está formada por varias capas de células epidérmicas. Una vaina de tejido conectivo formada por una capa circular de tejido conectivo denso y otra 


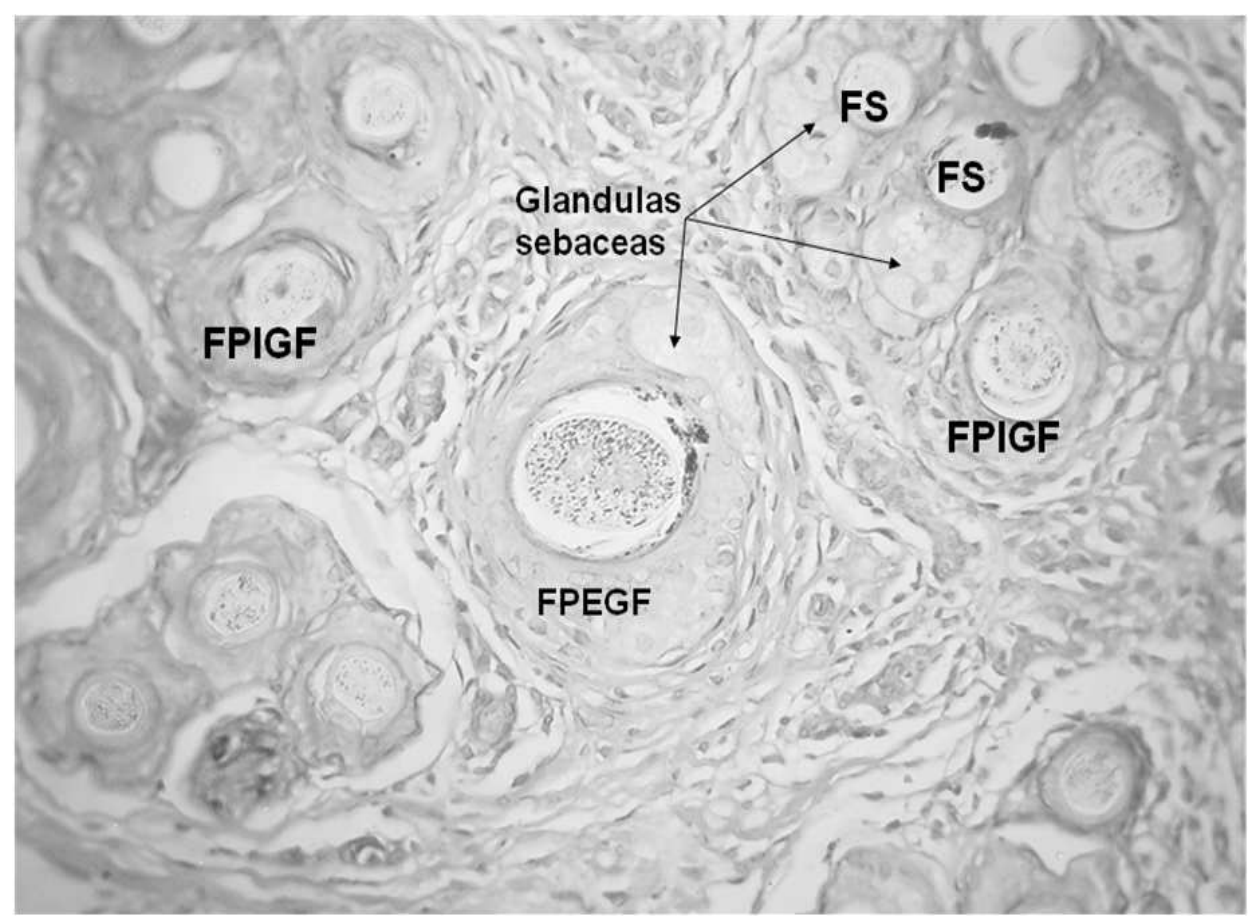

Figura 10. Distribución del folículo primario extra grupo folicular (FPEGF). Glándulas sebáceas asociadas al FPEGF y a los folículos secundarios (FS). FPIGF = Folículo primario intra grupo folicular. Coloración HE, $400 \mathrm{X}$

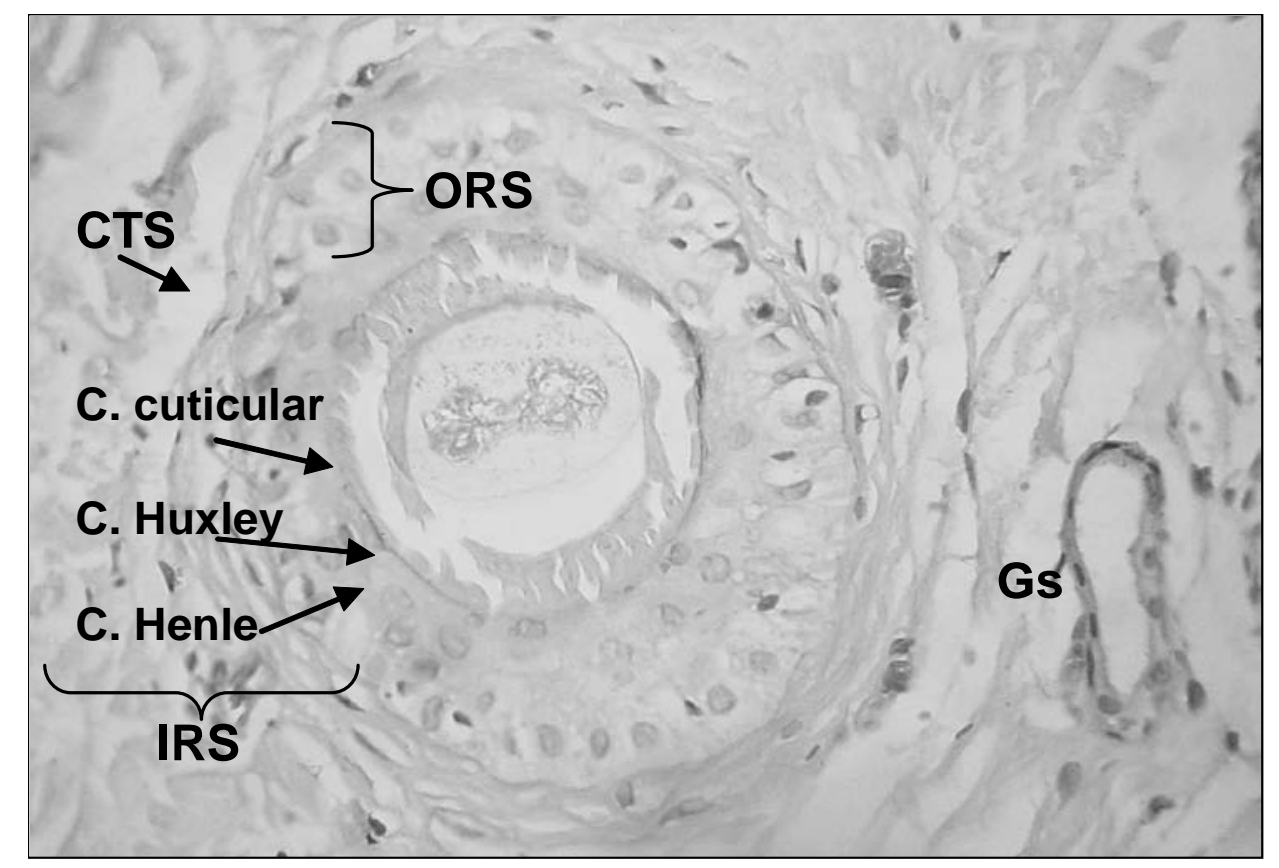

Figura 11. Estructura del folículo primario extra grupo folicular (FPEGF) con su pelo medulado en alpaca Huacaya. Vaina de la raiz interna (IRS) con tres subcapas (Henle, Huxley, Cuticular); Vaina de la raiz externa (ORS); Vaina de Tejido conjuntivo (CTS); Glándula sudoripara (Gs). Coloración HE, $400 X$ 
longitudinal externa que se presenta solamente en determinadas porciones rodeando a los vasos sanguíneos y conducto de glándulas sudoríparas. Por lo tanto, la diferencia entre los folículos primarios extra grupo folicular y el intra grupo folicular reside en la morfología de la médula y en el número de capas de la VRE.

\section{Discusión}

El estudio histológico de la piel del presente trabajo evidenció la presencia de dos tipos de folículo primario, el FP extra grupo folicular(FPEGF) y el FP intra grupo folicular (FPIGF). El primero de ellos, con una distribución en la piel de crías de alpaca, de manera similar a lo descrito por Rodríguez et al. (1985) en alpacas adultas, por Mamani (1988) en fetos de alpacas, y por Atlee et al. (1997) y Chambilla (1983) en llamas; pero erróneamente denominado como folículo simple, debido a que está conformado por una única estructura; mientras otros autores como Gaitán (1967) y Tapia $(1969,1977)$ no llegaron a evidenciar estas estructuras en alpacas adultas.

En este estudio se demuestra un patrón de medulación arriñonado, diferente con relación a la forma y diámetro de lo reportado en 1lamas (Chambilla, 1983) y alpacas (Rodríguez et al., 1985) donde se describen formas medulares en hélice incompleta y trébol. El diámetro medular del pelo de la alpaca es semejante a lo señalado por Rodríguez et al., (1985) quienes sostienen que es más pequeño que el de llama, lo cual es también señalado por Fowler (1989). Estos hallazgos confirman una superior calidad del pelaje de la alpaca en comparación con el de la llama. Asimismo, el FPEGF conforma un folículo piloso simple, completamente diferenciado, con abundante tejido conectivo que lo rodea, por encontrarse asociado con estructuras glandulares que pueden ser sebáceas o sudoríparas, coincidiendo con lo descrito en llamas por Chambilla (1983).
La distribución del folículo compuesto descrita por Calhoun y Stinson, (1986) en carnívoros domésticos como arreglos de un folículo primario simple rodeado por varios folículos secundarios no coincide con lo observado en este trabajo, donde el folículo simple (FPEGF) se encuentra en la periferia del grupo folicular compuesto, el cual está conformado por folículos primarios intra grupo foliculares (FPIGF) y folículos secundarios, tal y como se ha descrito en la llama (Atlee et al., 1997). Asimismo, se evidencia la presencia de grupos foliculares compuestos sin folículos primarios y conformados solo por folículos secundarios como ha sido descrito en estudios sobre crías de llamas (Copana et $a l ., 2000)$. Sin embargo, para una mejor definición histológica y ante estos hallazgos, se ha optado, por conveniente, la denominación de "Grupos foliculares simples". No obstante, es importante señalar que otros estudios realizados en alpacas adultas no mencionan la existencia de grupos foliculares carentes de folículos primarios (Gaitán, 1967; Tapia, 1969, 1977).

La conformación del grupo folicular por un FP y un número variable de FS, ha sido descrito por otros autores en alpacas (Gaitán, 1967; Tapia, 1969, 1977); asimismo, la presencia del trío folicular primario en alpacas Suri coincide con hallazgos reportados en llamas (Copana et al., 2000; Frank et al., 2006), y en alguna forma en ovinos merinos australianos (Carter y Clarke, 1957). Sin embargo, el hallazgo de un FP central de mayor diámetro que los primarios laterales y su asociación a un conducto sudoríparo difiere de la descripción dada por Rogers (2006) en ovinos donde los FP tienen el mismo diámetro dentro del grupo folicular en trío y, además, acompañados de una glándula sebácea multilobulada. Por otro lado, se encontró cierta variación en el número de FS que acompañan al FP dentro de un GFC, siendo de 3 a 25 FS por cada primario en alpacas Huacaya y de 3 a 20 en alpacas Suri, pero, en general, se puede decir que son bastante más numerosos que en la llama donde se han encontrado entre 3 a 10 FS por cada FP (Chambilla, 1983; Atlee et al., 1997). 
Los folículos pilosos se pueden encontrar asociados a glándulas sebáceas, las cuales son pequeñas en tamaño y generalmente unilobuladas (Gaitán, 1967; Tapia, 1969; Rodríguez et al., 1985); sin embargo, la piel de las alpacas Suri se caracterizan por presentar mayor cantidad de glándulas sebáceas asociadas a los folículos secundarios (Tapia, 1969), lo cual explicaría la lustrosidad y mayor peso de vellón en comparación con las alpacas Huacaya. Las glándulas sebáceas, en este tipo de animal cumpliría, además, una función protectiva debido a que sus fibras, por ser lacias y largas, cuelgan como mechas y se organizan a vellón abierto.

La existencia de manojos o haces de folículos secundarios que salen de un mismo orificio reportado en alpacas Huacaya (Gaitán, 1967) coincide con los resultados del presente estudio, donde se evidencian penachos de fibras pero emergentes de FS secundarios.

\section{Conclusiones}

- La diversa y compleja citoarquitectura del folículo piloso y su relación con las glándulas anexas y tejido conectivo subyacente permite establecer la presencia de un "Complejo Folicular Piloso" en las alpacas.

- Los folículos pilosos en las alpacas forman grupos foliculares compuestos y grupos foliculares simples.

- Los folículos primarios son de dos clases: el folículo primario solitario denominado folículo primario extra grupo folicular (FPEGF) y el folículo primario rodeado de varios folículos secundarios dentro de un grupo folicular compuesto denominado folículo primario intra grupo folicular (FPIGF).

- Las alpacas Suri presentan grupos foliculares compuestos por un folículo primario central y dos laterales.

\section{Agradecimientos}

Los autores agradecen al Dr. César Montalvo, Profesor Emérito, y a los doctores Alfonso Chavera y Rosa Perales, miembros del laboratorio de Histología, Embriología y Patología Veterinaria de la Facultad de Medicina Veterinaria de la Universidad Nacional Mayor de San Marcos, por su colaboración para el desarrollo del presente trabajo.

\section{Literatura Citada}

1. Atlee BA, Stannard A, Fowler ME, Olivry T. 1997. The histology of normal llama skin. Vet Dermatol 8: 165-176.

2. Banks WJ. 1993. Histología veterinaria aplicada. $3^{\mathrm{a}}$ ed. México DF: Ed. Manual Moderno. 698 p.

3. Calhoun ML, Stinson AW. 1986. Integument. En: Dellmann HD, Brown MB, eds. Histología Veterinaria. 3 ed. Zaragoza, España: Ed. Acribia. p 485500.

4. Carter HB, Clarke WH. 1957. The hair follicle group and skin follicle population of Australian Merino sheep. Austr J Agric Res 8(1): 91-108.

5. Chambilla V. 1983. Estructura histológica de la piel de llama (Lama glama). Tesis de Médico Veterinario. Puno: Facultad de Medicina Veterinaria y Zootecnia, Univ. Nacional del Altiplano. 45 p.

6. Charry AA. 1998. Soft rolling skin: is it a watershed for alpaca production. EFFN News 4: 8-12.

7. Copana C, Rodríguez T, Ayala C, Antonini M. 2000. Estructura y desarrollo de la población folicular de llamas en crecimiento. En: Libro Resúmenes III Congreso Mundial sobre Camélidos Sudamericanos. Potosí, Bolivia. p 627-632.

8. Fowler ME. 1989. Medicine and surgery of South American camelids. Ames: Iowa State University Press. 391 p.

9. Frank E, Hick M, Pesarini M. 2006. Un nuevo enfoque para la descripción 
histológica del complejo folicular epitelial en llamas argentinas. En: Camélidos sudamericanos domésticos: investigaciones recientes. Lima: DESCO. p. 78-88.

10. Gaitán DM. 1967. Estudio preliminar de los folículos pilosos en alpacas variedad Huacaya. Tesis de Ingeniero Zootecnista. Lima: Univ. Nacional Agraria La Molina. $31 \mathrm{p}$.

11. Junqueira LC, Carneiro J. 1988. Histología básica. $3^{a}$ ed. España: Ed. Salvat. $544 \mathrm{p}$.

12. Mamani FR. 1988. Estudio preliminar del desarrollo embriológico de la fibra en la piel de alpacas (Lama pacos). Tesis de Médico Veterinario. Puno: Facultad de Medicina Veterinaria y Zootecnia, Univ. Nacional del Altiplano. 40 p.
13. Rodríguez CC, Chambilla FV, Bustinza CV. 1985. Histología de la piel de alpaca y llama. Puno: Universidad Nacional del Altiplano.

14. Rogers GE. 2006. Biology of the wool follicle: an excursion into a unique tissue interaction system waiting to be rediscovered. Exp Dermatol 15:931-936.

15. Tapia CM. 1969. Estudio preliminar de la densidad y relación folicular de la piel de alpacas de la variedad Suri. Tesis de Ingeniero Zootecnista. Lima: Univ. Nacional Agraria La Molina. $31 \mathrm{p}$.

16. Tapia IM. 1977. Determinación de la estructura folicular en piel de alpaca. Tesis de Médico Veterinario. Puno: Facultad de Medicina Veterinaria y Zootecnia, Univ. Nacional del Altiplano. 66 p. 